(C) 2017 IEEE. Personal use of this material is permitted. Permission from IEEE must be obtained for all other uses, in any current or future media, including reprinting/republishing this material for advertising or promotional purposes, creating new collective works, for resale or redistribution to servers or lists, or reuse of any copyrighted component of this work in other works. 


\title{
Underwater mobile target tracking with particle filter using an autonomous vehicle
}

\author{
I. Masmitja ${ }^{1}$, P. J. Bouvet ${ }^{2}$, S. Gomariz ${ }^{1}$, J. Aguzzi ${ }^{3}$, J. del Rio ${ }^{1}$ \\ ${ }^{1}$ SARTI Research Group. Electronics Department, Universitat Politècnica de Catalunya. Vilanova i la Geltrú, Spain \\ ${ }^{2}$ Underwater Acoustics Lab, ISEN Brest. C.S. 42807, 29228 Brest cedex 2, France \\ ${ }^{3}$ Marine Science Institute (ICM). Consejo Superior de Investigaciones Científica (CSIC). Barcelona, Spain \\ Email: ivan.masmitja@upc.edu,pierre-jean.bouvet@isen-ouest.yncrea.fr
}

\begin{abstract}
This paper describes an underwater mobile target localization and tracking by using an autonomous surface vehicle for which the successive ranges between the target and the reference are the only information. In a dynamic system, such as range-only single-beacon underwater target tracking, a statespace model can be characterized, where the state vector may include position, and velocity of the mobile underwater target. Moreover, the range observations can come from a mobile autonomous vehicle, which is used as a moving landmark. Then, a nonlinear Bayesian filtering algorithm can be used to make extrapolations on the state vector from the observations, in order to obtain the target position at each instant of time. In this paper we consider the use of Particle Filter (PF) to perform such localization and tracking where its performance and characterization is studied under different scenarios.
\end{abstract}

Keywords- range-only; single-beacon; particle filter; autonomous vehicle; $A U V$; underwater positioning; underwater tracking

\section{INTRODUCTION}

Since its appearance two decades ago, the Particle Filter (PF) [1] has become one of the main techniques for the nonlinear Bayesian filtering problem, and nowadays numerous successful applications can be observed in literature, which cover different areas, form imaging processing [9] or terrestrial radar tracking [10], to underwater positioning.

Focusing on this last area, some papers, such as [2] and [3], have presented their work on target tracking, using both Time Difference Of Arrival (TDOA) and Time Of Arrival (TOA), and using a set of transponders as landmarks nodes. On the other hand, [4] and [5] have used PF for target tracking using Direction Of Arrival (DOA) or angle-only observations. Other authors, such as [6], have derived different methods to improve the PF performances, for example by introducing Gaussian Mixture Range-Only Filter (GMROF) algorithm.

However, most of these works use fixed transponders, where the main application is for radar detection and tracking [10]. Only few papers related to moving sensors (on autonomous vehicles), and for underwater applications can be found. For example, in [7] the authors use four Gliders for maritime vehicle tracking, and in [8] a procedure for animal tracking with an Autonomous Underwater Vehicle (AUV) is proposed.

In particular, this latter work has the same aim which is presented in this paper. However, while in [8] a method for animal tagged tracking and following is developed, by an array of two hydrophones attached on an AUV (and therefore they use the bearing measurements), we study the case where both the target and the vehicle are equipped with a very basic acoustic transponder providing only range information between target and vehicle. Moreover, we propose a complete study including performance characterization of the system and derivation of optimum values (such as speed ratio and range frequency).

Paper is organized as follows: section 2 is devoted to the description of studied tracking algorithms for range-only underwater positioning including Extended Kalman Filter (EKF) and PF based approaches while section 3 provides simulation results comparison and analysis between these 2 algorithms. Finally conclusions are drawn in section 4 .

\section{TRACKING ALGORITHMS}

The EKF [13], and its derivations such as Unscented Kalman Filter (UKF) [19], is one of the most widely used methods to solve the target position when both range and bearing information are available, or for multiple sensor systems. However, these methods can be useless in other situations, such as in range-only and single-beacon techniques.

The main problem of target tracking using a single sensor and using only ranges (or bearings) is the multimodal posterior probability distribution function of the system, which can be unobservable for a static sensor scenario.

Different methods can be used to solve this problem. For example, to find the correct target position at each time-step, a methodology which relies on the knowledge of the accurate dynamic model of the target. If this dynamic model is not available, both range and bearing information are required.

Other methods such as a group of filters running in parallel to track multiple hypothesis of the target's state have been used to solve this problem. For example, in the Range-Parametrized Extended Kalman Filter (RP-EKF) [11], each filter tracks one state in a specific interval of range, and finally a cost function is derived to find the best track.

On the other hand, the scientific community has paid attention to the Sequential Monte Carlo Methods (SMCM) such as PF for its capability of solving nonlinear estimation problems with multimodal pdf. Such as in [8].

Finally, in order to solve the unobservability problems, other authors have focused on observer maneuvers, for example, in 
[12] and the references therein. These studies examine the cases for which maneuvers of the observer are necessary and/or sufficient to obtain observability for target tracking. In general, the main concepts are non-collinear points and circumference paths shapes.

In this paper we compare the EKF and PF in a range-only single-beacon system, with an observer's maneuvers to ensure observability.

\section{A. Extended Kalman Filter (EKF)}

In this section we will use the standard notation to describe the main equations of an EKF. Our approach is to consider a single sensor (an autonomous vehicle) moving on the sea surface and estimating the state (position and velocity) of a moving target with a known depth, by processing the available range measurements.

The state vector of the target at time-step $k$ is defined by

$$
\mathbf{x}_{k}=\left[\begin{array}{llll}
x_{T k} & \dot{x}_{T k} & y_{T k} & \dot{y}_{T k}
\end{array}\right]^{T}
$$

where $x_{T k}$ and $y_{T k}$ are the target position in east-north coordinates, and $\dot{x}_{T k}$ and $\dot{y}_{T k}$ are their velocities.

In general, most authors consider the case where the target moves randomly, but assuming a constant acceleration or constant velocity, with the motion model

$$
\mathbf{x}_{k}=\mathbf{F}_{k-1} \mathbf{x}_{k-1}+\mathbf{Q}_{k-1}
$$

where $\mathbf{F}$ is the state transition matrix, and $\mathbf{Q}$ is the process noise with variance $\sigma_{v}^{2}$ related to time-step $\Delta t$, both described as

$$
\begin{gathered}
\mathbf{F}=\left[\begin{array}{cccc}
1 & \Delta t & 0 & 0 \\
0 & 1 & 0 & 0 \\
0 & 0 & 1 & \Delta t \\
0 & 0 & 0 & 1
\end{array}\right] \\
\mathbf{Q}=\left[\begin{array}{cccc}
\frac{1}{4} \Delta t^{4} & \frac{1}{2} \Delta t^{3} & 0 & 0 \\
\frac{1}{2} \Delta t^{3} & \Delta t^{2} & 0 & 0 \\
0 & 0 & \frac{1}{4} \Delta t^{4} & \frac{1}{2} \Delta t^{3} \\
0 & 0 & \frac{1}{2} \Delta t^{3} & \Delta t^{2}
\end{array}\right] \sigma_{v}^{2}
\end{gathered}
$$

On the other hand, the measurement model used in the rangeonly technique at time-step $k$ can be described by

$$
\begin{aligned}
h\left(\mathbf{x}_{k}\right) & =\left\|\mathbf{P}_{T k}-\mathbf{P}_{S k}\right\|+w_{k}= \\
& =\sqrt{\left(x_{T k}-x_{S k}\right)^{2}+\left(y_{T k}-y_{S k}\right)^{2}}+w_{k}
\end{aligned}
$$

where $\mathrm{P}_{T k}$ and $\mathrm{P}_{S k}$ are respectively the target and source position whereas $w_{k} \sim \mathcal{N}\left(0, \sigma_{w k}^{2}\right)$ is a zero-mean white Gaussian measurement noise with variance $\sigma_{w k}^{2}$, leading to a covariance matrix $\mathbf{R}=\operatorname{diag}\left[\sigma_{w k}^{2}\right]$. The Jacobian matrix of $h\left(\mathbf{x}_{k}\right)$ is computed as:

$$
\mathrm{H}=\left.\frac{\partial h\left(\mathbf{x}_{k}\right)}{\partial \mathbf{x}}\right|_{x}=\left[\begin{array}{c}
\frac{\left(x_{T}-x_{S}\right)}{\sqrt{\left(x_{T}-x_{S}\right)^{2}+\left(y_{T}-y_{S}\right)^{2}}} \\
0 \\
\frac{\left(y_{T}-y_{S}\right)}{\sqrt{\left(x_{T}-x_{S}\right)^{2}+\left(y_{T}-y_{S}\right)^{2}}} \\
0
\end{array}\right]
$$

\section{B. Particle Filter (PF)}

The main idea behind PF is the use of grids to represent the state space, and the posterior computation over this grid recursively. However, as a difference with Point Mass Filter (PMF) [14], the PF uses an adaptive stochastic grid which automatically selects the most relevant gird points in the state space, which are called particles, where each particle has its own state vector associated. This method reduces the computational complexity compared to PMF algorithms.

The algorithm's steps are described below, which follow the notation of [8] and [17]

1. Initialization: initialize the state vector (1) for each particle and its weight associated.

$$
\begin{aligned}
& \left\{\mathbf{x}_{0}^{n}\right\}_{n=1}^{N} \sim p\left(x_{0}\right) \\
& \left\{W_{0}^{n}\right\}_{n=1}^{N}=1 / N_{p}
\end{aligned}
$$

For time-step $k$ do:

2. Prediction: propagate particles according to motion model (2)

$$
\begin{array}{ll}
\hat{\mathbf{x}}_{k}^{n} \sim p\left(\mathbf{x}_{k} \mid \mathbf{x}_{k-1}^{n}\right), & \mathrm{n}=1, \ldots, N \\
\hat{\mathbf{x}}_{k}^{n}=\mathbf{F} \hat{\mathbf{x}}_{k-1}^{n}+\mathbf{Q}_{k-1}, & \mathrm{n}=1, \ldots, N
\end{array}
$$

3. Update: importance weight update using the measurement model (5)

$$
W_{k}^{n}=p\left(\mathrm{z}_{k} \mid \hat{\mathbf{x}}_{k}^{n}\right), \quad n=1, \ldots, N
$$

which are given by evaluating the likelihood function

$$
W_{k}^{n}=p\left(\mathrm{z}_{k}-h\left(\hat{\mathbf{x}}_{k}^{n}\right)\right), \quad n=1, \ldots, N
$$

4. Normalize the importance weights.

$$
W_{k}^{n}=W_{k}^{n} / \sum_{j=1}^{N} W_{k}^{j}, \quad n=1, \ldots, N
$$

5. Resampling: Generate a set of new particles from the previous set, according to the importance weights calculated in the previous step.

6. Repeat steps 2 to 5

where $n$ is the index of each particle in the PF, and $W^{n}$ is its importance weight associated.

The resampling step in a PF is an important process which has to be taken into account. This phase can introduce an important source of error related to randomness. Moreover, it can increase significantly the computational costs. The stratified and systematic sampling techniques reduce the variance of the system while using an efficient implementation scheme. 
Therefore, these are the most implemented methods for resampling in particle filters, see [15] and [16], and the references therein for more information.

\section{SIMULATIONS}

Both EKF and PF methods have been evaluated under different scenarios to validate their potentiality as a range-only single-beacon target positioning, tracking and monitoring systems. In Fig. 1 the generic scenario is represented, where a surface vehicle (the red triangle) follows an underwater target (the dark blue triangle) using only the computed target prediction: by $\overline{E K F}$ (the big light blue triangle) and $\overline{P F}$ (the big light pink triangle). The surface vehicle can be a Wave Glider (WG), which has a good qualities for this purpose including low power consumption, GPS-based localization, long deployment and good communication capabilities. The use of WG for static target localization has been experimented in [18] by conducting different missions in Monterey Bay and on the Californian coast.

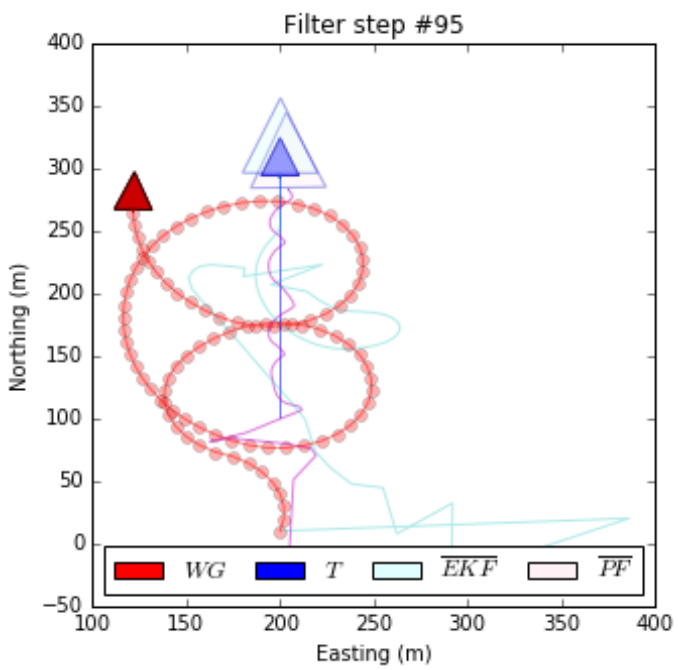

Fig. 1. Example of EKF and PF with range-only single-beacon target tracking and monitoring. The dark blue triangle is the underwater target, the red triangle is the autonomous surface vehicle, and the light blue and light pink are the estimated underwater target positions.

All the simulations have been done with Python by using parameters summarized in Table I.

TABLE I. FILTER CHARACTERISTICS

\begin{tabular}{|l|c|}
\hline \multicolumn{1}{|c|}{ Parameter } & Value \\
\hline WG initial position $[\mathrm{x}, \mathrm{y}, \mathrm{yaw}]$ & {$[300 \mathrm{~m}, 0 \mathrm{~m}, \pi / 2 \mathrm{rad}]$} \\
\hline Target initial position $[\mathrm{x}, \mathrm{y}, \mathrm{yaw}]$ & {$[200 \mathrm{~m}, 100 \mathrm{~m}, \pi / 2 \mathrm{rad}]$} \\
\hline WG velocity & $1 \mathrm{~m} / \mathrm{s}$ \\
\hline Target velocity & $0.2 \mathrm{~m} / \mathrm{s}$ \\
\hline Range's standard deviation $(\sigma)$ & $5 \mathrm{~m}$ \\
\hline Uncertainty covariance & 1000 \\
\hline Time between iterations & $10 \mathrm{~s}$ \\
\hline Ranging interval time & $10 \mathrm{~s}$ \\
\hline
\end{tabular}

\begin{tabular}{|c|c|}
\hline Parameter & Value \\
\hline Number of paricles & 3000 \\
\hline
\end{tabular}

\section{A. Setting time}

A first simulation was done with the parameters of Table I to observe the Root Mean Square Error (RMSE) in localization forboth algorithms. One of the most interesting parameters to characterize the spatiotemporal status of the tracked object is the setting time $T_{s}$, or the time that the algorithm need to find the true target's position. In this case, we have chosen an error of 15 $\mathrm{m}$ as a lower bound. The result can be observed in Fig. 2, where we obtained a $T_{s}=2$ minutes for the $P F$ and a $T_{s}=$ 13 minutes for the EKF algorithms. We can observe that the PF provides a much lower convergence time than EKF. Nevertheless, when the EKF has converged, the localization estimation is more accurate than the PF one.

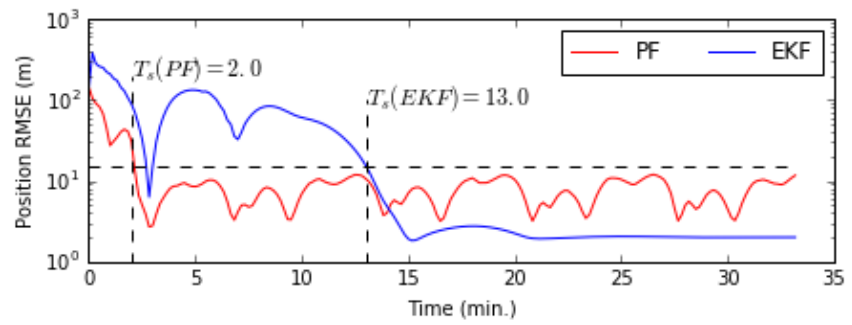

Fig. 2. Convergence time for both EKF and PF algorithms. Where the y-axis correspond to the Root-Mean-Square Error (RMSE) for $\mathrm{x}$ and $\mathrm{y}$ positions between the estimation and true target position.

Finally, two different scenarios have been simulated to observe the performance of the system under different conditions, which are speed ratio and range measurement frequencies.

\section{B. Speed ratio}

In this section, we study the performance of both filters at different speed ratio between the underwater target and the Wave Glider defined as

$$
r=\frac{v_{W G}}{v_{T}}
$$

where $v_{W G}$ and $v_{T}$ are the velocity of the $\mathrm{WG}$ and the underwater target respectively.

The results obtained are shown in Fig. 3. This test was conducted with the simulation parameters of Table I, excepted for the target velocity, which was related to the ratios $r=$ $[1,2,3,4,5,6,7,8,9,10]$. The 10 overlaid results are represented in the following plots.

One can observe that the velocity ratio between WG and Target have an important implication in the system's performance. If both velocities are similar, the algorithm cannot estimate correctly the target position. Therefore, this sets an important limitation as can be observed in Table II. For example, if we want a RMSE less than 10 meters, a speed ratio greater than 4 must be used (with the PF algorithm). 
TABLE II. SPEED RATIO RESULTS

\begin{tabular}{|c|c|c|}
\hline \multirow{2}{*}{ Speed ratio (r) } & \multicolumn{2}{|c|}{ RMSE $^{\mathbf{a}}$} \\
\cline { 2 - 3 } & $\boldsymbol{P}$ & $\boldsymbol{E} \boldsymbol{K} \boldsymbol{F}$ \\
\hline 1 & $249.07 \mathrm{~m}$ & $1869.74 \mathrm{~m}$ \\
\hline 2 & $37.91 \mathrm{~m}$ & $3.92 \mathrm{~m}$ \\
\hline 3 & $14.20 \mathrm{~m}$ & $3.51 \mathrm{~m}$ \\
\hline 4 & $9.99 \mathrm{~m}$ & $2.52 \mathrm{~m}$ \\
\hline 5 & $9.03 \mathrm{~m}$ & $2.02 \mathrm{~m}$ \\
\hline 6 & $6.83 \mathrm{~m}$ & $1.67 \mathrm{~m}$ \\
\hline 7 & $5.69 \mathrm{~m}$ & $1.43 \mathrm{~m}$ \\
\hline 8 & $4.99 \mathrm{~m}$ & $1.25 \mathrm{~m}$ \\
\hline 9 & $4.57 \mathrm{~m}$ & $1.11 \mathrm{~m}$ \\
\hline 10 & $4.02 \mathrm{~m}$ & $1.00 \mathrm{~m}$ \\
\hline
\end{tabular}
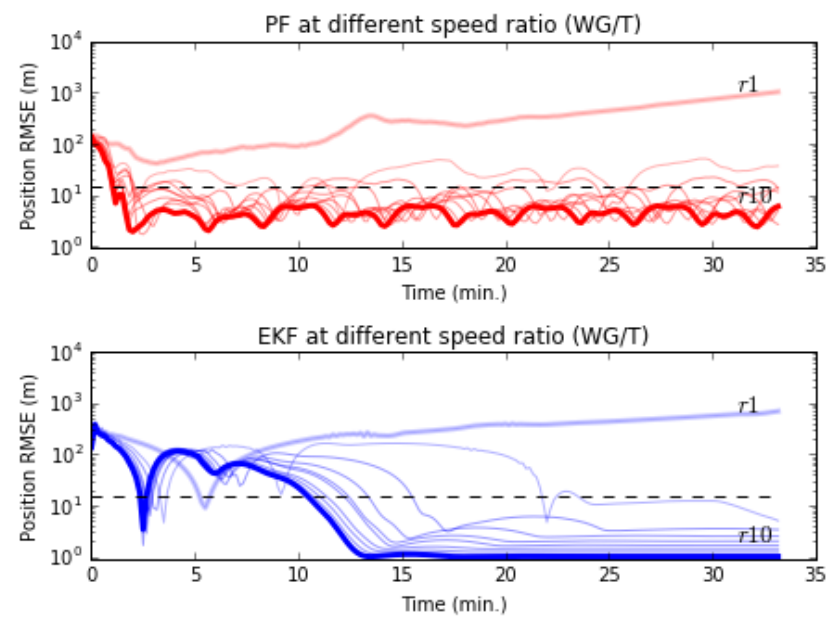

Fig. 3. Speed ratio effect for PF and EKF algorithms. Where the y-axis correspond to the Root-Mean-Square Error (RMSE) for $\mathrm{x}$ and $\mathrm{y}$ positions between the estimation and true target position.

In Table II, one can also observe that for a speed ratio equal to 1 neither the PF nor the EKF can converge to the true target position leading to very high an increasing RMSE.

\section{Ranging interval time}

The second parameter under study is the ranging interval time which represents the time between two successive range measurements. These intervals were set to $t=$ $[10,20,30,40,50,60,70,80,90,100]$ seconds.

The result obtained is shown in Fig. 4. This test was also done with the simulation parameters of Table I, except for the Ranging interval time. Again, we can see all 10 results overlaid for both PF and EKF.

Obviously, we can observe that performance of both algorithms increase as ranging interval time decreases. However, we can notice that both filters are less sensitive to this effect compared to the speed ratio explained above.
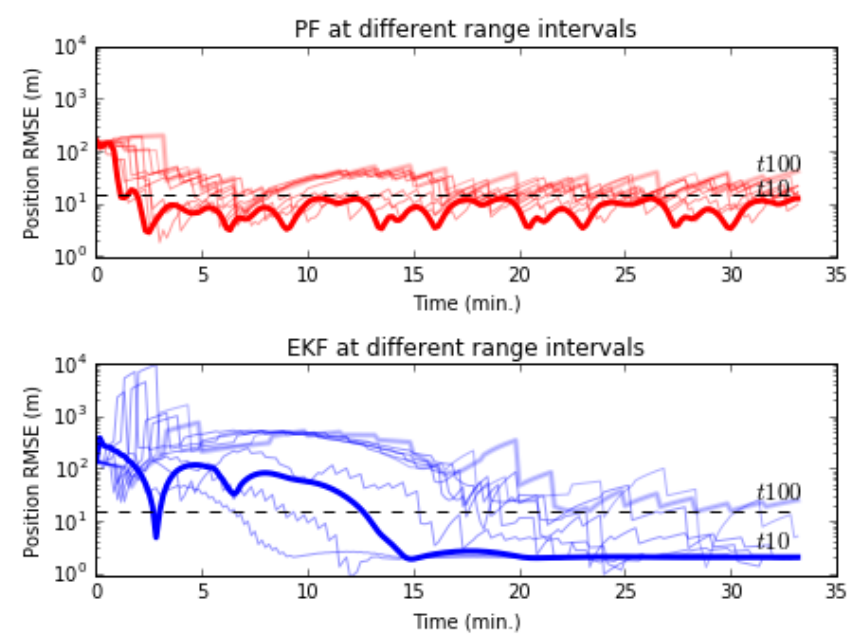

Fig. 4. New range's frequency effect for PF and EKF algorithms. Where the $\mathrm{y}$-axis corresponds to the Root-Mean-Square Error (RMSE) for $\mathrm{x}$ and $\mathrm{y}$ positions between the estimation and true target position.

\section{CONCLUSIONS}

The range-only single-beacon algorithms for underwater target positioning are interesting methods, which can be useful for many applications.

In this paper we follow such approach to localize and track an underwater target from a manoeuver surface vehicle by using EKF and PF filtering algorithms. Moreover, a study of different scenarios has been conducted, showing some bounds related to the speed ratio between observer and target and also the ranging interval time. The PF algorithms is demonstrated to achieve accurate localization estimation with fast convergence time.

Whereas the PF has a good setting time, the EKF is shown to provide a better estimation in a stationary state. Thus, in a future work, EKF and PF could be efficiently combined to provide both fast convergence and accurate localization.

\section{ACKNOWLEDGMENT}

This work was partially supported by the project NeXOS from the European Union's Seventh Programme for research, technological development and demonstration under the grant agreement No 614102. We also had financial support from the Spanish Ministerio de Economía y Competitividad under contract CGL2013- 42557-R (Interoperabilidad e instrumentación de plataformas autónomas marinas para la monitorización sísmica, INTMARSIS) and CTM2015-68804REDT (Instrumentation and Applied Technology for the Study, Characterization and Sustainable Exploration of Marine Environment, MarInTech). The main author of this work has a grant (FPI-UPC) from UPC for his PhD research.

\section{REFERENCES}

[1] F. Gustafsson, "Particle filter theory and practice with positioning applications," in IEEE Aerospace and Electronic Systems Magazine, vol. 25 , no. 7, pp. 53-82, July 2010. doi: 10.1109/MAES.2010.5546308 
[2] D. Carevic, "Localisation and tracking of underwater acoustic source using a modified particle filter," OCEANS 2010 IEEE - Sydney, Sydney, NSW, 2010, pp. 1-7.doi: 10.1109/OCEANSSYD.2010.5603865

[3] C. Jing, Z. Lin and J. Li, "Detection and tracking of an underwater target using the combination of a particle filter and track-before-detect," OCEANS 2016 - Shanghai, Shanghai, 2016, pp. 1-5. doi: 10.1109/OCEANSAP.2016.7485684

[4] X. Zhong, A. B. Premkumar and W. Wang, "Direction of arrival tracking of an underwater acoustic source using particle filtering: Real data experiments," IEEE 2013 Tencon - Spring, Sydney, NSW, 2013, pp. 420424. doi: 10.1109/TENCONSpring.2013.6584482

[5] Jiaxiang Yu, Deyun Xiao and Xiuting Yang, "Square Root Unscented Particle Filter with Application to Angle-Only Tracking," 2006 6th World Congress on Intelligent Control and Automation, Dalian, 2006, pp. 15481552. doi: 10.1109/WCICA.2006.1712610

[6] J. M. C. Clark, P. A. Kountouriotis and R. B. Vinter, "A Gaussian Mixture Filter for Range-Only Tracking," in IEEE Transactions on Automatic Control, vol. 56, no. 3, pp. 602-613, March 2011. doi: 10.1109/TAC.2010.2072590

[7] I. Kyriakides, E. Abdi and D. Hayes, "Maritime vehicle tracking using underwater gliders and particle filters," 2016 4th International Workshop on Compressed Sensing Theory and its Applications to Radar, Sonar and Remote Sensing (CoSeRa), Aachen, Germany, 2016, pp. 51-55.

[8] C. M. Clark, C. Forney, E. Manii, D. Shinzaki, C. Gage, M. Farris, C. G. Lowe, and M. Moline, (2013), "Tracking and Following a Tagged Leopard Shark with an Autonomous Underwater Vehicle". J. Field Robotics, 30: 309-322. doi:10.1002/rob.21450

[9] Lu, W-L., Okuma, K., and Little, J. "Tracking and recognizing actions of multiple hockey players using the boosted particle filter'. Image and Vision Computing, 27 (2009), 1 89-205.

[10] V. Cevher, R. Velmurugan and J. H. McClellan, "A Range-Only Multiple Target Particle Filter Tracker," 2006 IEEE International Conference on
Acoustics Speech and Signal Processing Proceedings, Toulouse, 2006, pp. IV-IV. doi: 10.1109/ICASSP.2006.1661116

[11] N. Peach, MA, C. Eng, "Bearings-only tracking using a set of rangeparameterised extended Kalman filters" Control Theory and Applications, IEE Proceedings, vol 142, Issue 1, pp.73 - 80, Jan 1995

[12] D. Moreno-Salinas, N. Crasta, M. Ribeiro, B. Bayat, A.M. Pascoal, J. Aranda, "Integrated Motion Planning, Control, and Estimation for RangeBased Marine Vehicle Positioning and Target Localization," IFACPapersOnLine, Volume 49, Issue 23, 2016, Pages 34-40, ISSN 24058963, http://dx.doi.org/10.1016/j.ifacol.2016.10.318.

[13] A. Jazwinski. "Stochastic processes and filtering theory". Academic Press, 1970

[14] N. Bergman, "Recursive Bayesian Estimation: Navigation and Tracking Applications," PhD. Thesis, 1999

[15] S. Thrun, W. Burgard, D. Fox, "Probabilistic robotics," MIT Press, Cambridge, MA, 2005, pp. 67-90

[16] J. Fernández-Madrigal and J. L. Blanco Claraco, "Simultaneous Localization and Mapping for Mobile Robots: Introduction and Methods," 2013. (pp. 1-499). Hershey, PA: IGI Global. doi:10.4018/9781-4666-2104-6

[17] Thomas B. Schon, "Solving nonlinear state estimation problems using particle filters--an engineering perspective," 2010. Technical Report. Automatic Control at Linkopings Universitet.

[18] I. Masmitja, S. Gomariz, J. Del Rio, B. Kieft and T. O'Reilly, "Rangeonly underwater target localization: Path characterization," OCEANS 2016 MTS/IEEE Monterey, Monterey, CA, 2016, pp. 1-7. doi: 10.1109/OCEANS.2016.77612

[19] S. J. Julier and J. K. Uhlmann, "A New Extension of the Kalman Filter to Nonlinear Systems," in Proc. of AeroSense: The 11th Int. Symp. on Aerospace/Defence Sensing, Simulation and Controls., 1997. 